\section{Prótesis mucosoportada en la rehabilitación de un paciente totalmente edéntulo con fisura palatina}

\author{
Muco supported prosthesis in the rehabilitation of a totally edentulous \\ patient with cleft palate
}

\title{
Resumen
}

La rehabilitación protésica de pacientes con paladar fisurado y completamente desdentados requiere, entre otros, la preparación de una prótesis con obturador. El principal objetivo del tratamiento es sustituir las estructuras ausentes y restablecer las funciones masticatoria, deglutiva, y del habla. En este artículo se presenta la rehabilitación con prótesis completa y obturador hueco, de un paciente con fisura palatina de aproximadamente $3 \mathrm{~cm}$, que comunica la cavidad oral con la nasal sin involucrar el paladar blando. Se describen los aspectos clínicos y de laboratorio para la preparación de la aparatología con la que se logró restablecer las funciones perdidas, el sellado de la comunicación buco nasal, resultando de gran beneficio para la deglución, la dicción y la recuperación del aspecto psicológico.

Palabras clave: paladar fisurado; obturador palatino; prótesis completa.

\section{Abstract}

The prosthetic rehabilitation of patients with cleft palate and completely toothless requires, among others, the preparation of a prosthesis with shutter. The main goal of treatment is to replace the missing structures and restore masticatory, swallow and speech functions. This article presents the rehabilitation of a patient with cleft palate of approximately $3 \mathrm{~cm}$, connecting the oral and nasal cavities without involving the soft palate. It is described, clinical and laboratory aspects for the preparation of the appliance with which it was possible to restore the lost functions, sealing the buco nasal communication, resulting in great benefit for swallowing, speech and psychological recovery aspect.

Keywords: cleft palate; palatal obturator; total prosthesis.

1. Unidad de Posgrado de la Facultad de Odontología de la Universidad Nacional Mayor de San Marcos, Perú.

Haydeé Luján-Larreategui', Martha Pineda-Mejia ${ }^{1}$

\section{Introducción}

Las fisuras labio palatinas son malformaciones craneofaciales congénitas que en nuestro medio se presenta en una proporción del 1 por cada 1000 nacidos, siendo la frecuencia mayor en hombres $(54.55 \%)$ que en mujeres $(45.5 \%)^{1}$, se debe a la falta de unión entre algunos procesos faciales embrionarios que dan origen a estas estructuras. Muchas de estas anomalías congénitas parecen determinadas genéticamente mientras que la mayoría son de causa desconocida o son atribuibles a causas teratógenas ${ }^{2}$.

El labio y el paladar se forman durante el desarrollo embrionario de la sexta a la novena semana de vida intrauterina. El paladar primario se desarrolla a partir de la prolongación palatina medial y da origen a la premaxila. El paladar secundario se desarrolla a partir de las prolongaciones palatinas laterales del proceso frontonasal que se fusiona al tabique nasal ${ }^{3}$.
Las malformaciones por labio y paladar hendidos causan alteraciones funcionales importantes como son el paso de alimentos y líquidos de la cavidad oral hacia la cavidad nasal con el riesgo inherente de asfixia, paso de fluidos nasales hacia la cavidad bucal, pérdida de resonancia en la emisión del habla con dificultad para la pronunciación de ciertos fonemas debido a la hiper nasalidad, dificultad en la masticación por pérdida de estructura maxilar incluyendo los dientes, dificultad en la deglución por alteraciones del patrón de cierre velo faríngeo y alteraciones estéticas por pérdida de soporte de los tejidos blandos ${ }^{3,4,5}$.

La rehabilitación quirúrgica o protésica persigue los mismos objetivos: crear una separación física entre las cavidades oronasales u orosinusales así como restaurar el habla, la deglución, la masticación y dar soporte a los tejidos blandos, el labio y las mejillas ${ }^{6}$.

Lo ideal en cuanto a la rehabilitación protésica del paciente con labio y pa- ladar fisurado es pasar por tres etapas distintas en las cuales se usan distintos tipos de obturadores: a) Obturador quirúrgico o inmediato, b) Obturador temporal, c) Obturador definitivo el cual debe ser diseñado cuando los tejidos estén cicatrizados, no existan signos de recidiva de actividad neoplásica y no esté programada ni indicada una nueva intervención quirúrgica ${ }^{8,10}$.

Las prótesis obturadoras son dispositivos que reparan un defecto mediante la ocupación del espacio creado como consecuencia de la pérdida o ausencia de tejido en el maxilar superior.

Deben ser de bajo peso, estables, no irritantes, cómodas, de diseño sencillo, fáciles de movilizar y capaces de suplir tanto el contorno como las funciones fisiológicas perdidas. La rehabilitación protésica de un paciente portador de una fisura palatina y que a la vez es completamente desdentado requiere, entre otros, la preparación de una prótesis completa con obturador ${ }^{12,13,14}$. 
El objetivo del tratamiento debe ser sustituir las estructuras ausentes y restablecer las funciones masticatoria, deglutiva, y del habla. De acuerdo con Sharma ${ }^{9}$, los factores que contribuyen a la desestabilización de la prótesis son: a) reducido tamaño del defecto maxilar, b) espacio interoclusal demasiado grande, c) estructura ausente del paladar duro, d) cresta alveolar subdesarrollada con escasa profundidad palatina, e) cicatrización tras el cierre labial.

El objetivo de este trabajo es mostrar una alternativa de tratamiento de manera conservadora, a un paciente totalmente desdentado que presenta fisura palatina, que le permita restablecer las funciones de estética, fonética y masticación.

\section{Caso clínico}

Paciente masculino de 52 años de edad, completamente desdentado con fisura palatina que va desde la parte media del paladar duro hasta el paladar blando no llegando a dividir la úvula, no ha sido tratada quirúrgicamente (Fig.1), hay buena competencia labial y los rebordes alveolares presentan continuidad en su trayecto. Según informó el paciente acudió a varios odontólogos para rehabilitarse y mejorar su masticación y estética, pero no había obtenido resultados con los que se encontrara satisfecho, decidió intentar una vez más, es así que llega a la consulta.

A través de la anamnesis, se tomó conocimiento que la única intervención quirúrgica que tuvo fue para cerrar el labio leporino unilateral. Desde infante, fue portador de prótesis obturadoras, no refiere con exactitud la fecha de última prótesis realizada.

El paciente es portador de prótesis total superior e inferior, que se ven desadaptadas. Radiográficamente (Fig. 2.) se observa: en el maxilar superior, imagen radio opaca de límites continuos de mediano diámetro ubicado en reborde posterior de lado derecho, en el sector anterior del primer cuadrante se observa reabsorción severa de los rebordes alveolares, con falta de continuidad de las líneas radiopacas que corresponden al paladar. El maxilar inferior presenta rebordes alveolares con reabsorción moderada generalizada, las demás estructuras no presentan alteración.

Tratamiento: Se opta por realizar prótesis total de acrílico con obturador de fisura palatina.

\section{Impresiones:}

La toma de la impresión primaria para el maxilar superior se realizó con algi- nato y cubetas de stock, colocando una gasa en la zona de la fisura, para evitar que el material de impresión ingrese en la cavidad nasal (Fig.3, Fig.4). Se preparó el modelo primario con yeso piedra tipo III (Fig. 5) a partir del cual se elaboró la cubeta individual con resina de autocurado, teniendo la precaucion de bloquear las zonas retentivas alrededor de la fisura (Fig.6, Fig.7) y se alivió con cera calibrada toda la superficie del reborde. La cubeta no se introduce en el defecto.

\section{Impresión definitiva, sellado periférico y de los ángulos palatales:}

- Se recortó la cubeta a dos milímetros del fondo se surco para realizar el sellado periférico con godiva en lápiz, primero con calor seco y luego atemperamiento con agua tibia, (calor húmedo) para evitar producir quemaduras en la mucosa, se obtiene el sellado de todo el fondo de surco y además de los ángulos palatales, zona ubicada a nivel del paladar duro y paladar blando donde no hay hueso, solo mucosa y tejido blando, (Fig. 8).

- El siguiente paso fue la impresión definitiva con pasta zinquenólica (Fig.9) obteniendo la copia de toda la zona aprovechable del reborde.

- En éste procedimiento a diferencia de la impresión primaria, no se coloca la gasa para el bloqueo del defecto, el diseño de la cubeta copia el reborde y la zona del defecto, realizando movimientos funcionales.

- Determinación de la Dimensión Vertical: elaboración de la placa base y rodetes de oclusión y contorno, (Fig.10).

- Contorno facial, altura del rodete orientación en el espacio con el instrumento plano de Fox y los planos de Camper y bipupilar.

- Pruebas fonéticas (Ley de Pound) rodete superior pronunciación de letra "F" rodete inferior pronunciación letra "M"

- Dimensión Vertical.

- Registro en Céntrica con cera alumínica Aluwax (Fig.10)

- Alineamiento y articulado de dientes, seleccionando los dientes según forma, tamaño y color, luego de la cual se procedió a la prueba en paciente.
- Cirugía en modelo en los ángulos palatales, antes del procesado en la zona de los ángulos palatales (Es una técnica de desgaste en el modelo definitivo de yeso antes de enviar a procesar el caso clínico, ubicado en la zona del postdamming, límite de paladar duro y paladar blando) y tallado de retenciones en modelo definitivo a inicio de fisura palatina, (Fig.11)

- Instalación de prótesis completa con obturador, (Fig.12).

- Luego que el paciente recibiera instrucciones sobre la higiene bucal, se concertaron las visitas de control. En las visitas de control el paciente manifestó mejoras en el habla, masticación y confort. (Fig.13, Fig.14).

\section{Discusión}

Debido a las alteraciones de la cresta alveolar de las personas con labio leporino y fisura palatina, la rehabilitación con dentadura completa es un reto para el profesional, las dificultades se producen en relación a la estabilidad y la retención de la prótesis, ya que los factores físicos, tales como adhesión, tensión superficial y la cohesión están deteriorados ${ }^{7,14}$.

En este caso el paciente cuenta con una fisura palatina, sin fistulas, el reborde alveolar es continuo en la zona anterior, el paladar blando está completo y la úvula unida, lo cual nos hace presumir que la prótesis obturará la fisura palatina y controlará la filtración de aire o líquidos hacia la cavidad nasal, sin mayor dificultad.

Para los procedimientos a seguir en la preparación de la prótesis obturadora se tomó como referencia los criterios clínicos para la rehabilitación de un desdentado total normal.

Las modificaciones realizadas durante la toma de impresiones primaria y definitiva, permitieron tener un control del material de impresión para que no ingrese en grandes cantidades a la cavidad oronasal.

Para cubrir la fisura palatina se realizó la prótesis total con acrílico de uso frecuente, y se realizó la cirugía en modelo. Se realizaron retenciones en el modelo definitivo antes del procesado, en la zona de la fisura palatina.

Driscoll y Habib ${ }^{11}$ presentaron la idea de ahuecar el aditamento obturador para aligerar el peso de la prótesis optimizando su comodidad durante la 


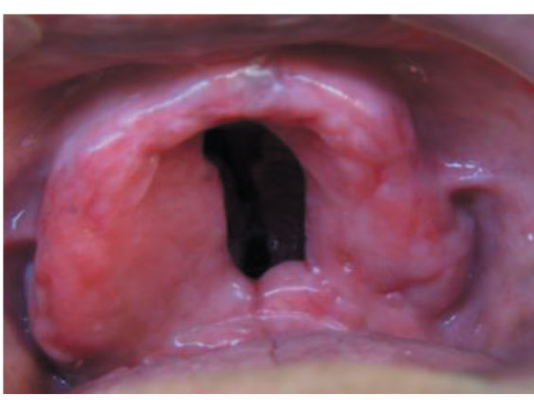

Fig.1. Fisura palatina y conservación de la úvula

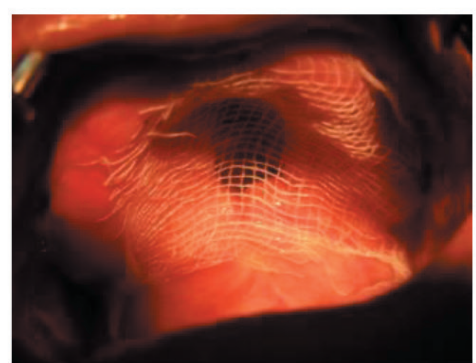

Fig.3. Bloqueo de la zona de fisura

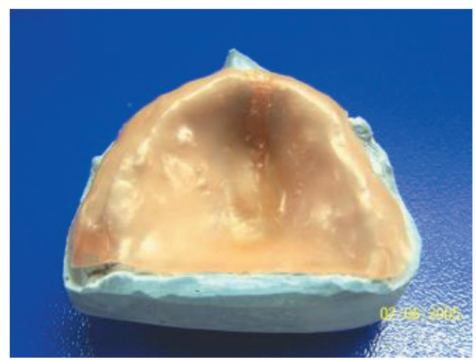

Fig. 6. Cubeta individual

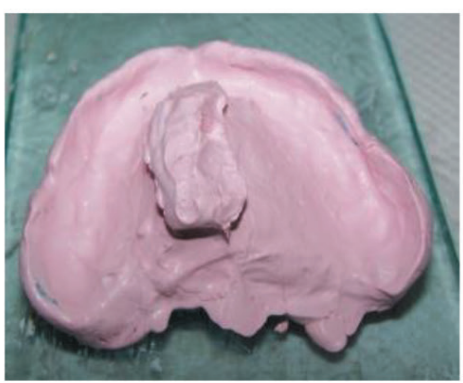

Fig. 9. Impresión definitiva

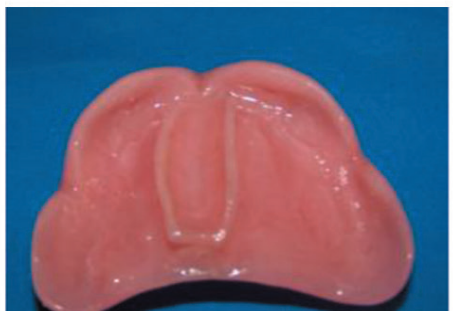

Fig.12. Prótesis y obturador para instalación

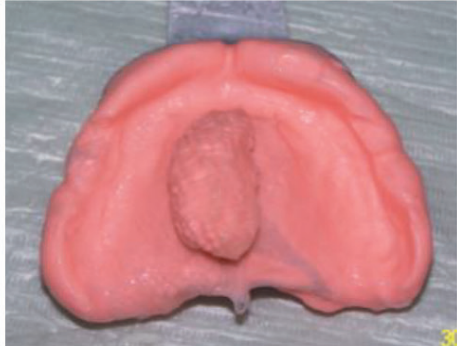

Fig.4. Impresión primaria y alivios

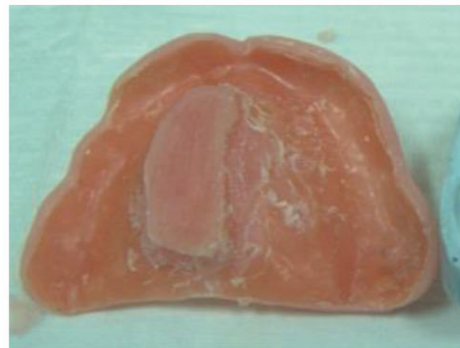

Fig.7. Cubeta individual

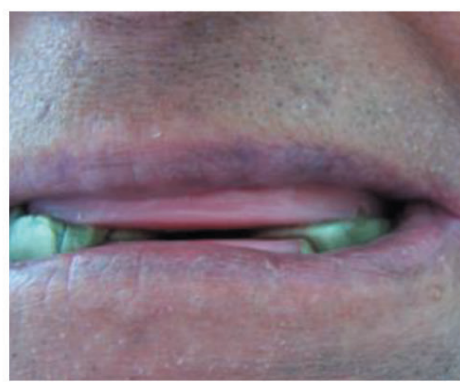

Fig. 10. Registro en céntrica

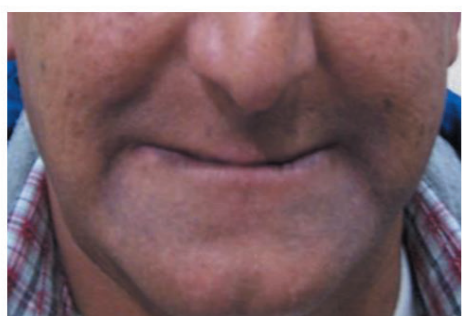

Fig.13. Caso antes

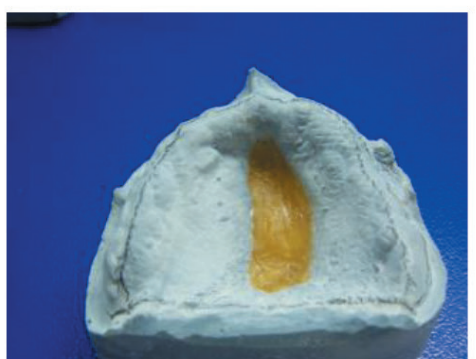

Fig.5.Modelo primario y alivio

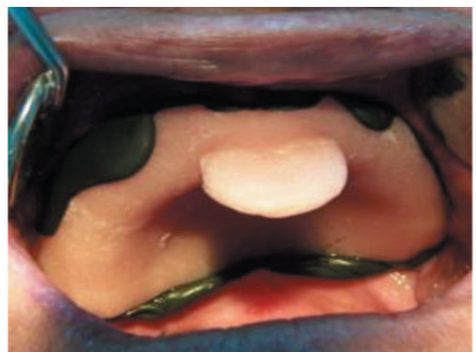

Fig.8. Copia de todo el reborde y zona de defecto

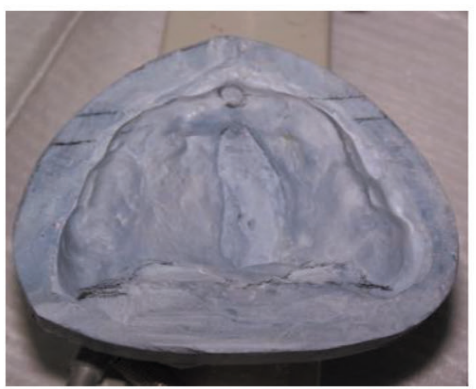

Fig. 11. Zona de cirugía del modelo definitivo

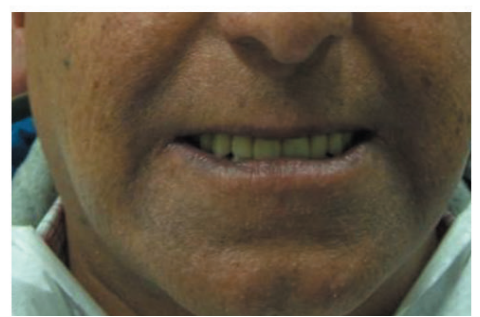

Fig.14. Caso después 
masticación, la fonación y la deglución, en caso de defectos extensos, lo cual fue tomado en cuenta para este caso, introduciéndose solo lo suficiente para sellar el defecto.

En la cita de entrega de la prótesis se aliviaron las zonas de presión.

La retención obtenida fue excelente, $y$ fue dada por el mismo defecto. El paciente, con este tratamiento, mejoró su confianza y sin duda alguna su calidad de vida, el habla, la fonética, la deglución, la masticación y su estética fueron notablemente mejoradas.

\section{Conclusiones}

La rehabilitación de pacientes con secuelas de labio leporino y paladar fisurado todavía sigue siendo un reto para los profesionales de la salud, ya que siempre se requiere de la preparación de prótesis no convencionales individualizadas.

Cuando la comunicación oro-nasal no sea demasiado extensa y el defecto no presente retenciones anatómicas, los obturadores huecos ofrecen ventajas, por su mayor estabilidad y menor peso.

Con este tipo de prótesis maxilofacial obturamos la comunicación oro-nasal en su totalidad, mejorando la fonación, deglución y el estatus psicológico, relaciones interpersonales, en suma su calidad de vida.

\section{Referencias bibliográficas}

1. Sacsaquispe S, Ortiz L. Prevalencia de labio y/o paladar fisurado y factores de riesgo. Rev. Estomatol Herediana 2004;14(1-2):54-58.

2. Mc Donald RE, Avery DR. Odontología pediátrica y del adolescente. 5a ed. Argentina: Medica Panamericana, 1991. 848p.

3. Donado RM. Cirugía bucal. $3^{\mathrm{a}}$ ed. Espańa: Masson, 2005: 618-627.

4. Beumer J, Curtis TA, Marunick. Maxillofacial rehabilitation. Prosthodontic and surgical considerations. Canada: Euroamerica, 1996.

5. Corbo RMT, Marimón TME. Labio y paladar fisurados. Aspectos generales que se deben conocer en la atencion primaria de salud. $\mathrm{Rev} \mathrm{Cu}-$ bana Med Gen Integr, 2001, 17(4).

6. Torres TF, Marín LC, Jiménez CR, Alvarado GE, Uribe QE. Elaboración de una sobredentadura modificada para paciente con secuelas quirúrgicas de labio y paladar hendidos: reporte de un caso. Rev. Odont. Mex. 2013 jul sep:17(3).

7. De Souza FJA, Fraga de Almeida ALP, Soares S, Teixeira das Neves L, Gamba GD. Rehabilitative treatment of cleft lip and palate: experience of the Hospital for Re- habilitation of Craniofacial Anomalies/USP (HRAC/USP). Part 4: Oral Rehabilitation. J Appl Oral Sci. 2012;20(1):9-15.

8. Salinas-González JS, Diaz-Aguirre CM, Echevarria y-Perez E, Hernández-Cuéllar A. Evaluacion functional y estetica de obturadores palatines en pacientes maxilectomizados. GAMO, 2011;10(5):278-287.

9. Sharma AB, Beumer J. Reconstruction of maxillary defects: The case for prosthetic rehabilitation. J Oral Maxillofac Surg 2005;63:1770-3.

10. Ono T, Kohda H, Hori K, Nokubi T. Masticatory performance in postmaxillectomy patients with edentulous maxillae fitted with obturator prostheses. Int $\mathrm{J}$ Prosthodont 2007;20(2):145-150.

11. Driscoll CF, Habib BH. Fabrication of a closed hollow obturator. J Prosthet Dent. 2004;91:383-5.

12. 1B. Koeck. Protesis completas. $4^{\mathrm{a}}$ ed. Barcelona: Elsevier-Masson; 2007. 408p.

13. Fischer H, Gutowsky A. et. al. Bases de la prótesis dental total. Mexico: Ediciones Doyma; 1999.

14. Ferreira S. Rehabilitación bucal odontopediatrica. Caracas: Amolca; 2003. 